\title{
Homocysteine, antioxidant vitamins and lipids as biomarkers of neurodegeneration in Alzheimer's disease versus non-Alzheimer's dementia
}

\author{
Grzegorz Raszewski', Roman Chwedorowicz², Agnieszka Chwedorowicz², \\ Katarzyna Gustaw Rothenberg ${ }^{2,3}$ \\ ${ }^{1}$ Department of Physiopathology, Institute of Rural Health, Lublin, Poland \\ ${ }^{2}$ Department of Neurodegenerative Diseases, Institute of Rural Health, Lublin, Poland \\ ${ }^{3}$ Department of Psychiatry Yale University School of Medicine, New Haven, USA
}

Raszewski G, Chwedorowicz R, Chwedorowicz A, Gustaw Rothenberg K. Homocysteine, antioxidant vitamins and lipids as biomarkers of neurodegeneration in Alzheimer's disease versus non-Alzheimer's dementi. Ann Agric Environ Med. 2016; 23(1): $193-196$. doi: $10.5604 / 12321966.1196878$

\begin{abstract}
I Abstract
Introduction and objective. Evidence for the benefit of antioxidants' based therapeutic intervention in dementia are inconsistent. Parallel studies in disease forms of dementia different than Alzheimer's are even less conclusive. In this study, the role of serum levels of homocysteine (tHcy), lipids and antioxidants in predicting the risk of cognitive decline in Alzheimer's disease (AD) versus non-Alzheimer's dementias ( $n-A D)$. The objective was to add to the ongoing cumulative research to establish the biochemical baseline for potential nutri-therapeutic intervention in different forms of dementia.

Materials and method. 65 participants with dementia (DP-s) were divided into two groups: ADP - patients with Alzheimer's disease and $n-A D P$ - patients with dementia of a different etiology than primary neurodegenerative dementia in the course of Alzheimer's disease. Cognitive function was assessed by Mini-Mental State Examination (MMSE) and related to plasma levels of tHcy, folate, vitamins B-6, B-12, lipids and vitamins A and E for both groups. Also examined were associations between cognitive impairment and several variables (age, education, duration of dementia) that might confound nutrition-cognition associations.

Results. A significant reduction in serum vitamin A levels and elevation of total cholesterol levels were shown for the DP-s group compared to those in the control group. Moreover, significant differences were found in MMSE data and serum vitamin $E$ and tHcy levels between patients with ADP and $n-A D P$. The scores for MMSE showed a correlation with the vitamin $E$ levels and duration of dementia in the ADP group and/or correlation with tHcy, levels of vitamins A and/or E, and duration of dementia in the n-ADP group.

Conclusions. The results obtained suggest that elevated serum tHcy and decreased levels of vitamins $\mathrm{A}$ and $\mathrm{E}$ are associated with an increased risk of non-Alzheimer's dementias, although further studies involving a larger cohort are now needed to verify these results.
\end{abstract}

\section{Key words}

cognitive decline, Alzheimer's disease, non-Alzheimer's dementias, homocysteine, antioxidant vitamins, lipids

\section{INTRODUCTION}

Dementia, more precisely defined as significant cognitive impairment, is not a specific disease but a group of different entities. It is a descriptive term for a collection of symptoms that can be caused by a number of disorders that affect the brain. The most common type of dementia is Alzheimer's disease (AD) which makes up $50 \%-70 \%$ of cases. Other common types include vascular dementia (25\%), Lewy body dementia (15\%), and frontotemporal dementia. Less common causes include normal pressure hydrocephalus, Parkinson's disease, neurosyphilis, and Creutzfeldt-Jakob disease among others [1]. AD leads to a progressive loss of mental functions through a very specific type of neurodegeneration. Other types of dementia may vary, however, in the character of progressive changes. All the above-mentioned diseases share not only a main symptomatology of cognitive decline, but also may share some of the risk factors.

Address for correspondence: Grzegorz Raszewski, Department of Physiopathology Institute of Rural Health, Jaczewskiego 2, 20-090 Lublin, Poland

e-mail: raszewskigj@gmail.com

Received: 29 April 2015; accepted: 18 September 2015
Some evidence suggests that an increased production of reactive oxygen species and/or a decreased efficiency of antioxidant defence systems are associated with aging. Oxidative metabolic reactions and their by-products have been consistently implicated in the pathogenesis of agerelated dementia and represent the biological basis for the 'oxidative stress hypothesis' of $\mathrm{AD}$ [2]. The brain is particularly susceptible to the attack of free radicals caused by its low content of antioxidants, by the considerable content of lipids, and by its high oxygen consumption rate [3]. The braincell mitochondria are both a source and a target of reactive oxygen species (ROS). Therefore, mitochondrial dysfunction and oxidative metabolism may play an important role in the pathogenesis of $\mathrm{AD}$ and other age-related dementias. Moreover, the accumulation of ROS in neurons in neurons over time could account for the late-life onset and gradually progressive nature of the decline in dementia patients (4). In mammalian model systems, the effect of oxidative stress on lifespan is less clear, but there is evidence that antioxidant treatment protects against age-related dysfunction, including cognitive decline [4].

An elevated serum homocysteine (tHcy) level has proved not only to be an independent risk factor for both vascular 
dementia (VaD) and $\mathrm{AD}[5]$, but the one with significant strength. Furthermore, several epidemiological studies have assessed the link between lipids levels and dementia, especially AD, but cross-sectional studies provided mutually exclusive results and thus highly conflicting conclusions [6].

The objective of this study was to examine the role of serum levels of tHcy and its biological determinants, plasma lipids and antioxidants as predictors of cognitive decline in $\mathrm{AD}$, compared to different form of dementia.

\section{MATERIALS AND METHOD}

The participants were randomly selected from a populationbased sample within the Lublin Region. After screening and neuropsychiatric diagnostic procedures, 64 patients with different type of dementia (DP-s) were recruited to participate in the study. Dementia was defined on the basis of the clinical criteria of DSM-IV (Diagnostic and Statistical Manual of Mental Disorders, $4^{\text {th }}$ edition) [7]. AD was diagnosed on the basis of NINCDS-ADRDA (National Institute of Neurological and Communicative Disease and Stroke-Alzheimer's Disease and Related Disorders Association) criteria for probable or possible AD [8]. The Mini-Mental State Examination (MMSE) [9] was used for cognitive screening. Subjects with an MMSE scores $<26$ were considered positive at screening.

DP-s $(n=64)$ were divided into 2 groups: patients with AD (ADP, Group I; $n=33$ ) and patients with n-AD (n-ADP, Group II; $n=31$ ). The exclusion criteria consisted of untreated or not fully controlled hypercholesterolaemia, hypertension, diabetes, as well as other metabolic syndromes. Major psychiatric other than cognitive illnesses also excluded participant. The control group comprised 40 elderly subjects with no signs of dementia: 22 women and 18 men, mean age $73.6(\mathrm{SD} \pm 9.4)$.

All participants were provided with and signed a consent form prior to participation in the study. The research protocol was approved by the Ethics Committee of the Institute of Rural Health in Lublin, Poland.

Sample Collection. After an overnight fast, blood samples were obtained from the peripheral vein puncture following a 15-min rest. $5 \mathrm{ml}$ of blood was taken from each subject for analysis Within $1 \mathrm{~h}$ of the extraction, samples were collected in dry tubes for separating serum. Serum samples were separated by centrifugation at 3,000 rpm during a period of $15 \mathrm{~min}$. Serum for lipid analyses was refrigerated at $4{ }^{\circ} \mathrm{C}$ and assayed within 8 hours. Other serum samples were frozen and stored at $-81^{\circ} \mathrm{C}$ until analysis.

Assay Procedure. Baseline tHcy in serum was measured by using an adaptation of the high-performance liquid chromatography (HPLC) method described by Chwatko and Bald [10] with a Zorbax SB-C-18 column (4.6x150 mm, $5 \mu)$. Vitamins $A$ and $\mathrm{E}$ were detected, after extraction with ethanol and hexane, by HPLC with UV detection according to the recommended procedure for a commercial kit from Immuniq (Immundiagnostik AG, Germany). Baseline serum vitamin B-6, vitamin B-12 and folate (folic acid) were detected by using a direct MPT assay kits (Immundiagnostik AG, Germany).

Serum biochemical variables were determined in an Express Plus autoanalyzer (Chiron Diagnostics System). In this analysis, total cholesterol (TCh), triglycerides (TG) and HDL-cholesterol (HDL-C) were examined by colourimetric methods (Siemens Diagnostics, Mannheim, Germany). The concentration of LDL-cholesterol (LDL-C) was calculated using the Friedewald formula. Coefficients of variation (CVs) for all analyses in this study were $3.0-9.2 \%$.

Statistics. Data were expressed as the mean \pm standard deviation (SD) or median (range) according to data distribution. Comparisons between groups were performed using a one-way ANOVA test. Univariate correlations between the MMSE scores and other selected variables were analyzed using the non-parametric Spearman correlation coefficient, according to data distribution. $\mathrm{P}<0.05$ was considered significant. The statistical analyses were carried out using Graph-Pad Prism-5 for Windows.

\section{RESULTS}

A total of 64 patients with clinically diagnosed dementia provided samples for the evaluation. The biometrics or the DP, and the control are shown in Table 1. Of these, 23 cases (35\%) were male and 42 cases $(65 \%)$ were female. The mean age of the patients was 74.3 years $(\mathrm{SD} \pm 7.6)$, with an age range of $56-87$ years. Of these 64 subjects, 31 were diagnosed as $\mathrm{AD}$, and 33 diagnosed with other forms of dementia and were considered as one group, distinct from the $\mathrm{AD}$ group, for this purpose. Core statistical analysis included a healthy, age matched control as a comparator for both study groups.

Table 1. Characteristics of participating patients with dementia and controls

\begin{tabular}{lccc}
\hline Variable & $\begin{array}{c}\text { Dementia patients } \\
\mathrm{n}=64\end{array}$ & $\begin{array}{c}\text { Controls } \\
\mathrm{n}=40\end{array}$ & $\mathrm{p}=$ \\
\hline Age, years & $74.3 \pm 7.6$ & $73.6 \pm 9.4$ & 0.254 \\
\hline Duration of education, years & $9.9 \pm 3.3$ & $10.4 \pm 3.1$ & 0.773 \\
\hline Duration of dementia, years & $6.7 \pm 2.2$ & - & - \\
\hline MMSE scores (max.30) & $15.3 \pm 5.7$ & - & -
\end{tabular}

Serum variable:

\begin{tabular}{llll}
\hline Total cholesterol, mmol/I & $6.1 \pm 1.2$ & $5.6 \pm 1.1$ & 0.028 \\
\hline LDL cholesterol, mmol/I & $4.3 \pm 1.1$ & $4.1 \pm 1.1$ & 0.413 \\
\hline $\mathrm{HDL}$ cholesterol, $\mathrm{mmol} / \mathrm{I}$ & $1.2 \pm 0.3$ & $1.4 \pm 0.4$ & 0.547 \\
\hline Triglyceride, $\mathrm{mmol} / \mathrm{I}$ & $1.4 \pm 0.6$ & $1.2 \pm 0.6$ & 0.421 \\
\hline Total homocysteine, $\mu \mathrm{mol} / \mathrm{I}$ & $27.7 \pm 16.2$ & $21.3 \pm 13.6$ & 0.664 \\
\hline Vitamin B6, nmol/I & $79.5 \pm 109.1$ & $48.4 \pm 105$ & 0.344 \\
\hline Vitamin B12, pmol/l & $336.8 \pm 198.8$ & $304 \pm 226$ & 0.496 \\
\hline Folate, $\mathrm{nmol} / \mathrm{I}$ & $27.4 \pm 18.9$ & $28.0 \pm 17.7$ & 0.547 \\
\hline Vitamin A, $\mu \mathrm{mol} / \mathrm{I}$ & $3.8 \pm 0.1$ & $4.2 \pm 0.2$ & 0.034 \\
\hline Vitamin $\mathrm{E}, \mu \mathrm{mol} / \mathrm{I}$ & $35.8 \pm 6.3$ & $38.7 \pm 7.3$ & 0.057 \\
\hline
\end{tabular}

HDL - high-density lipoprotein; LDL - low-density lipoprotein; MMSE - Mini-Mental State Examination; Mean \pm Standard Deviation, $p$-value (level of significance).

As per objective of the protocol, the patients did not differ in age. Additionally, no significant dementia patients and the controls did not differ significantly when the level of education was considered. The serum concentrations of TCh and Vitamin A levels, however, were found to be significantly different between the DP-s and controls $(\mathrm{p}=0.028 ; \mathrm{p}=0.034$, respectively). 
Further evaluation focused of both dementia samples revealed that the ADP group had a significantly lower serum tHct and vitamin E level, compared to the n-ADP group $(\mathrm{p}=0.041 ; \mathrm{p}=0.014$, respectively) (Tab. 2$)$.

Table 2. Characteristics of participating patients with Alzheimer's disease (ADP-group) and non-Alzheimer dementia type (n-ADP-group)

\begin{tabular}{|c|c|c|c|}
\hline Variable & $\begin{array}{c}\text { ADP-group } \\
n=31\end{array}$ & $\begin{array}{c}\text { n-ADP-group } \\
n=33\end{array}$ & $p=$ \\
\hline Age (years) & $77.3 \pm 6.8$ & $71.1 \pm 7.2$ & 0.002 \\
\hline Duration of education (years & $10.0 \pm 3.3$ & $10.9 \pm 3.5$ & 0.199 \\
\hline Duration of dementia (years) & $6.7 \pm 2.3$ & $3.9 \pm 2.6$ & $<0.001$ \\
\hline MMSE scores (max. 30) & $15.3 \pm 5.7$ & $20.4 \pm 6.8$ & $<0.001$ \\
\hline \multicolumn{4}{|l|}{ Serum variable: } \\
\hline Total cholesterol, mmol/l & $6.1 \pm 1.2$ & $6.1 \pm 1.2$ & 0.683 \\
\hline LDL cholesterol, mmol/I & $4.2 \pm 1.1$ & $4.3 \pm 1.1$ & 0.992 \\
\hline HDL cholesterol, mmol/l & $1.1 \pm 0.3$ & $1.2 \pm 0.2$ & 0.141 \\
\hline Triglyceride, mmol/l & $1.3 \pm 0.5$ & $1.3 \pm 0.6$ & 0.847 \\
\hline Total homocysteine, $\mu \mathrm{mol} / \mathrm{l}$ & $24.2 \pm 15.5$ & $27.8 \pm 14.0$ & 0.041 \\
\hline Vitamin $\mathrm{B} 6, \mathrm{nmol} / \mathrm{l}$ & $43.6 \pm 79.1$ & $46.3 \pm 137$ & 0.176 \\
\hline Vitamin B12, pmol/l & $335 \pm 166$ & $371 \pm 226$ & 0.120 \\
\hline Folate, $\mathrm{nmol} / \mathrm{l}$ & $26.7 \pm 15.6$ & $28.7 \pm 21.9$ & 0.659 \\
\hline Vitamin A, $\mu \mathrm{mol} / \mathrm{I}$ & $3.6 \pm 0.9$ & $3.9 \pm 0.8$ & 0.124 \\
\hline Vitamin $E, \mu \mathrm{mol} / \mathrm{I}$ & $34.2 \pm 4.4$ & $37.6 \pm 7.5$ & 0.014 \\
\hline
\end{tabular}

HDL - high-density lipoprotein; LDL - low-density lipoprotein; MMSE - Mini-Mental State Examination; Mean \pm Standard Deviation, $p$-value (level of significance).

The mean age and duration of dementia for the $n$-ADP group were lower than for ADP-group $(\mathrm{p}=0.002 ; \mathrm{p}<0.001$, respectively (Tab. 2). Significant differences were also found in MMSE data $(\mathrm{p}<0.001)$ between patients with $\mathrm{AD}$ and $\mathrm{n}-\mathrm{AD}$.

The MMSE score was found to be inversely related to the duration of dementia $(r=-0.924, p<0.001 ; r=-0.897 ; p<0.001)$, respectively, for the ADP and $\mathrm{n}$-ADP groups and with age $(\mathrm{r}=-0.377 ; \mathrm{p}=0.037)$ for the $\mathrm{n}$-ADP group (Tab. 3 ).

Simple correlations between serum variables and MMSE scores are presented in Table 3.

Serum levels of vitamin E was found to be positively correlated with the MMSE score for both groups of DP-s: ADP-group $(r=0.346 ; p=0.049)$ and $n-A D P$ group $(r=0.488$; $\mathrm{p}=0.005)$ and inversely related to duration of dementia $(\mathrm{r}=-0.390, \mathrm{p}=0.024 ; \mathrm{r}=-0.511, \mathrm{p}=0.003)$, respectively. In addition, the serum concentration for vitamin A was found to be positively associated with the MMSE score of n-ADP group only $(\mathrm{r}=0.584 ; \mathrm{p}<0.001)$. Finally, the serum levels for Hct were found to be inversely correlated with the MMSE scores $(\mathrm{r}=-0.463 ; \mathrm{p}=0.009)$ in those patients.

Correlations of the serum concentrations for TCh, TG, HDL-C, vitamins B6, B12, foliate with MMSE scores failed to show any statistical significance (Tab. 3 ).

\section{DISCUSSION}

The results of the presented study show that cognitive decline was significantly associated with serum concentrations of vitamins A or E in patients with non-Alzheimer's dementia type disease. These findings supports the hypothesis that dietary interventions aimed at modifying serum vitamin E status may affect the risk of cognitive impairment in
Table 3. Association between MMSE scores and variables in Dementia patients

\begin{tabular}{lcccc}
\hline Variable & \multicolumn{3}{c}{ Correlation coefficient $(\mathrm{r}) \mathrm{a}$} \\
\hline & $\begin{array}{c}\text { ADP-group, } \\
\mathrm{n}=31\end{array}$ & $\mathrm{P}=$ & $\begin{array}{c}\mathrm{n} \text {-ADP-group, } \\
\mathrm{n}=33\end{array}$ & $\mathrm{P}=$ \\
\hline Age, years & -0.060 & 0.740 & -0.377 & $\mathbf{0 . 0 3 7}$ \\
\hline Duration of education, years & 0.214 & 0.232 & 0.072 & 0.700 \\
\hline Duration of dementia, years & -0.924 & $>0.001$ & -0.897 & $>0.001$ \\
\hline Serum variable: & & & & \\
\hline Total cholesterol, $\mathrm{mmol} / \mathrm{l}$ & -0.065 & 0.720 & -0.216 & 0.244 \\
\hline HDL cholesterol, $\mathrm{mmol} / \mathrm{l}$ & -0.122 & 0.498 & 0.140 & 0.453 \\
\hline Triglyceride, $\mathrm{mmol} / \mathrm{l}$ & 0.126 & 0.484 & 0.046 & 0.814 \\
\hline Homocysteine, $\mu \mathrm{mol} / \mathrm{l}$ & 0.084 & 0.644 & -0.463 & $\mathbf{0 . 0 0 9}$ \\
\hline Vitamin B6, $\mathrm{nmol} / \mathrm{l}$ & -0.147 & 0.416 & 0.185 & 0.319 \\
\hline Vitamin B12, $\mathrm{pmol} / \mathrm{l}$ & -0.065 & 0.719 & 0.260 & 0.158 \\
\hline Folate, $\mathrm{nmol} / \mathrm{l}$ & -0.033 & 0.856 & 0.169 & 0.363 \\
\hline Vitamin A, $\mu \mathrm{mol} / \mathrm{l}$ & 0.306 & 0.084 & 0.584 & $\mathbf{0 . 0 0 1}$ \\
\hline Vitamin $\mathrm{E}, \mu \mathrm{mol} / \mathrm{l}$ & 0.346 & $\mathbf{0 . 0 4 9}$ & 0.488 & $\mathbf{0 , 0 0 5}$ \\
\hline
\end{tabular}

a Pearson product moment correlation analysis with no adjustment

HDL, high-density lipoprotein; LDL, low-density lipoprotein; MMSE, Mini-Mental State Examination; p-value (level of significance)

older persons [11]. In addition, the Chianti Study [12] shows that in the elderly, both prevalence and incidence of cognitive impairments are associated with variations in blood concentrations of non-tocopherol forms of vitamin $\mathrm{E}$. However, the results of other studies on cognitive function and blood levels of vitamin E [13] and vitamin A [14] are inconsistent, and of even greater importantance, the supplementation did not prove to be of therapeutic value.

The current study also shows that MMSE score were inversely related to age and to duration of dementia. As expected, the significant differences in MMSE score in AD and $\mathrm{n}-\mathrm{AD}$ patients were probably caused the time of duration of disease. The mean duration of dementia in patients with $\mathrm{AD}$ and n-AD were 6, 7 and 3, 9 years, respectively. A significant negative relationship was found only between serum tHcy levels and MMSE scores in n-ADP. Other researchers have also shown a significant negative association between serum tHcy concentration and cognitive impairment tested by the MMSE score in patients with probable AD [15]. Although some studies found no association between plasma tHcy levels and cognitive impairment in patients with $\mathrm{AD}$ [16], in the presented study, the mean serum tHcy concentrations for $\mathrm{AD}$ and $\mathrm{n}-\mathrm{AD}$ patients were elevated in comparison to upper reference limits for tHcy $(20 \mu \mathrm{mol} / \mathrm{l})$ in patients aged $>65$ years, and in comparison to control, but no statistically significant differences were noted. However, it is worth noting that serum concentrations of folate and vitamins B-6, B-12 for these patients were within the normal range (Tab.1).

An elevated serum $\mathrm{tHcy}$ level might also suggest a vitamin B-12 deficiency. However, this indicator has poor specificity because it is influenced by other factors, such as low vitamin B6 or folate levels [16]. However, evidence suggests that serum vitamin B-12 concentrations might not accurately reflect intracellular concentrations [17]. Serum concentrations of vitamin B12 and folate in the current study were comparable to the reference range, but were inversely related to plasma tHcy levels $(r=-0.276 ; p=0.027$ and $r=-0.327 ; p=0.008$, respectively) (data not shown). 
In $\mathrm{n}$-ADP, a significant relationship was found between MMSE scores and serum levels of tHcy. Moreover, no correlation was observed with serum concentrations of tHcy and MMSE in ADP group. Therefore, based on the results of own studies, it might be presumed that elevated serum tHey may been associated with an increased risk of dementia of a non-Alzheimer's type.

A large prospective study showed that increased tHcy up to 11 years before the diagnosis of dementia is associated with twice the risk of AD. This study also showed positive associations between elevated tHcy levels and the incidence of both $\mathrm{AD}$ and dementia [18]. Moreover, a recent study indicated that homocysteine is an independent risk factor for both dementia and cognitive impairment [19]. Low serum levels of certain B vitamins (folate and vitamins B-6 and B-12) have been associated with elevated serum homocysteine levels in several studies, and with an increased risk of dementia in a many investigations. The results of these investigations show that elevated serum tHcy concentrations and low serum B vitamins concentrations are independent predictors of the development of dementia and AD [20].

The presented study indicates that serum lipid levels had no relationship with MMSE scores in ADP-s; however, the level of lipids in the blood serum was moderately elevated in this patient group.

Hypercholesterolaemia is a proven risk factor for cardiovascular disease [21] but the association with cognition appears complicated. The explanation for the conflicting epidemiologic associations between lipids and dementia is that other lipoprotein constituents may be more relevant in dementia pathology and may therefore represent a more accurate dementia risk factor. Apolipoprotein A-I (ApoA-I), a major component of HDL, may be such a factor [6]. In some studies, lower HDL-C levels have been associated with lower MMSE scores and with higher dementia and Alzheimer's disease risks $[22,23]$.

\section{CONCLUSIONS}

This study shows a significant relationship between cognitive impairment, tested by the MMSE score, and serum levels of antioxidant vitamins $\mathrm{A}, \mathrm{E}$ and $\mathrm{tHc}$ in patients with nonAlzheimer's type dementia.

Some limitations should be noted in the present study:

1) the association between the antioxidants status in patients with dementia and the risk of cognitive impairment was only cross-sectional; therefore, it was not possible to determine whether significant differences in plasma antioxidants in patients is a cause or consequence of cognitive impairment;

2) the findings from this study do not exclude the possibility that plasma antioxidant capacity may be modified by dietary factors;

3) other confounding factors (e.g. alcohol drinking, and smoking), which might affect the plasma antioxidant levels, were not completely excluded;

4) the sample size was relatively small for drawing a definite conclusion.

Despite multiple limiting factors listed above, the data obtained support the notion that elevated serum tHct levels, as well as vitamins A and E deficiencies, may be associated with an increased risk of non-Alzheimer's dementias. Further studies with a larger cohort are now needed to follow those threads and eventually find parameters for potentially effective pharmaceutical intervention.

\section{REFERENCES}

1.Ferri CP, Prince M, Brayne C, BrodatyH, Fratiglioni L, Ganguli M, et al. Global prevalence of dementia: a Delphi consensus study. Lancet 2005; 366: 2112-2117.

2. Pratico D. Oxidative stress hypothesis in Alzheimer's disease: a reappraisal. Trends Pharmacol Sci. 2008; 12: 609-615.

3. Savitha S, Tamilselvan J, Anusuyadevi M, Panneerselvam Ch. Oxidative stress on mitochondrial antioxidant defense system in the aging process: Role of dl-a-lipoic acid and l-carnitine. Clin Chim Acta. 2005; 355: $173-180$.

4. Evidence of oxidative stress in Alzheimer's disease brain and antioxidant therapy: lights and shadows. Ann N Y Acad Sci. 2008; 1147: 70-78.

5. Villa P, Bosco P, Ferri R, Perri C, Suriano R, Costantini B, et al. Fasting and post-methionine homocysteine levels in Alzheimers disease and vascular dementia Int J Vitam Nutr Res. 2009; 79(3): 166-172.

6. Panza F, D'Introno A, Colacicco AM. Lipid metabolism in cognitive decline and dementia. Brain Res Rev. 2006; 51: 275-292.

7. American Psychiatric Association. Diagnostic and statistical manual of mental disorders. 4th ed. Washington, DC, USA: American Psychiatric Association, 2000.

8. McKhann G, Drachman D, Folstein M, Katzman R, Price D, Stadlan EM. Clinical diagnosis of Alzheimer's disease: Report of the NINCDSADRDA work group under the auspices of department of health and human services task force on Alzheimer's disease. Neurology 1984; 34: 939-944.

9. Folstein MF, Folstein SE. "Mini-Mental State:" a practical method for grading the cognitive state of patients for the clinician. J Psychiatr Res. 1975; 2: 189-198.

10. Chwatko G, Bald E. Determination of different species of homocysteine in human plasma by high-performance liquid chromatography with ultraviolet detection. J Chromatogr A. 2002; 949: 141-151.

11. Dunn JE, Weintraub S, Stoddard AN, Banks S. Serum a-tocopherol, concurrent and past vitamin $\mathrm{E}$ intake, and mild cognitive impairment. Neurology 2007; 68: 670-676.

12. Cherubini A, Martin A, Andres-Lacueva C, Dilorio A, Lamponi M, Mecocci P. Vitamin E levels, cognitive impairment and dementia in older persons: the In CHIANTI study. Neurobiol Aging. 2005; 26 : 987-994.

13. Grodstein F, Chen J, Willet WC. High-dose antioxidant supplements and cognitive function in community-dwelling elderly women. Am J Clin Nutr. 2003; 77: 975-984.

14. Engelhart MJ, Ruitenberg A, Meijer J, Kiliaan A, van Swieten JC, Hofman A, et al. Plasma levels of antioxidants are not associated with Alzheimer's disease or cognitive decline. Dement Geriatr Cogn Disord. 2005; 19: 134-139.

15. Lepara O, Alajbegovic A, Zaciragic A, Nakas-Icindic E, Valjevac A, Lepara D, et al. Elevated serum homocysteine level is not associated with serum C-reactive protein in patients with probable Alzheimer's disease. J Neural Transm. 2009; 116(12): 1651-1656.

16. Li L, Cao D, Desmond R, Rahman A, Lah J, Levey A, et al. Cognitive Performance and Plasma Levels of Homocysteine, Vitamin B 12, Folate and Lipids in Patients with Alzheimer Disease. Dement Geriatr Cogn Disord. 2008; 26: 384-390.

17. Clarke R. B-vitamins and prevention of dementia. Proc Nutr Soc. 2008; 67: 75-81.

18. Annerbo S, Kivipelto M, Lökk J. A prospective study on the development of Alzheimer's disease with regard to thyroid-stimulating hormone and homocysteine. Dement Geriatr Cogn Disord. 2009; 28: 275-280.

19. Kalmijn S, Launer L, Lindemans J, Bots ML, Hofman A, Breteler MM. Total homocysteine and cognitive decline in a community-based sample of elderly subjects: the Rotterdam Study. Am J Epidemiol. 1999; 150: $283-289$.

20. Tucker KL, Qiao N, Scott T, Rosenberg I, Spiro A. High homocysteine and low $\mathrm{B}$ vitamins predict cognitive decline in aging men: the Veterans Affairs Normative Aging Study. Am J Clin Nutr. 2005; 82: 627-635.

21. Levine GN, Keaney JF, Vita JA. Cholesterol reduction in cardiovascular disease. Clinical benefits and possible mechanisms. N Engl J Med. 1995; 332: 512-521.

22.van Exel E, de Craen AJ, Gussekloo J, Houx P, Bootsma-van der Wiel A, Macfarlane PW. et al. Association between high-density lipoprotein and cognitive impairment in the oldest old. Ann Neurol. 2002; 51: 716-721.

23. Merched A, Xia Y, Visvikis S, Serot JM, Siest G. Decreased high-density lipoprotein cholesterol and serum apolipoprotein AI concentrations are highly correlated with the severity of Alzheimer's disease. Neurobiol Aging. 2000; 21: 27-3. 